\title{
gु
}

\section{Cooling by Heating: Very Hot Thermal Light Can Significantly Cool Quantum Systems}

\author{
A. Mari ${ }^{1,2}$ and J. Eisert ${ }^{1,2}$ \\ ${ }^{1}$ Dahlem Center for Complex Quantum Systems, Freie Universität Berlin, 14195 Berlin, Germany \\ ${ }^{2}$ Institute for Physics and Astronomy, University of Potsdam, 14476 Potsdam, Germany
}

(Received 11 April 2011; published 23 March 2012)

\begin{abstract}
We introduce the idea of actually cooling quantum systems by means of incoherent thermal light, hence giving rise to a counterintuitive mechanism of "cooling by heating." In this effect, the mere incoherent occupation of a quantum mechanical mode serves as a trigger to enhance the coupling between other modes. This notion of effectively rendering states more coherent by driving with incoherent thermal quantum noise is applied here to the optomechanical setting, where this effect occurs most naturally. We discuss two ways of describing this situation, one of them making use of stochastic sampling of Gaussian quantum states with respect to stationary classical stochastic processes. The potential of experimentally demonstrating this counterintuitive effect in optomechanical systems with present technology is sketched.

PACS numbers: 05.70.Ln, 42.50.Ar, 42.50.Lc, 85.85.+j
\end{abstract}

Cooling in quantum physics is usually achieved in just the same way as it occurs in classical physics or in common everyday situations: One brings a given system into contact with a colder bath. Coherent driving of quantum systems can effectively achieve the same aim, most prominently in instances of laser cooling of ions or in its optomechanical variant, cooling mechanical degrees of freedom using the radiation pressure of light. The coherence then serves a purpose of, in a way, rendering the state of the system "more quantum." In any case, in these situations, the interacting body should first and foremost be cold or coherent.

In this work, we introduce a paradigm in which thermal hot states of light can be used to significantly cool down a quantum system. To be specific, we will focus on an optomechanical [1-6] implementation of this idea: This type of system seems to be an ideal candidate to demonstrate this effect with present technology; it should however be clear that several other natural instances can well be conceived. Intuitively speaking, it is demonstrated that due to the driving with thermal noise, the interaction of other modes can be effectively enhanced, giving rise to a "transistorlike" effect [7]. We flesh out this effect at hand of two approaches following different approximation schemes. The first approach is essentially a weak coupling master equation, while the second approach makes use of stochastic samplings with respect to colored classical stochastic processes [9], which constitutes an interesting and practical tool to study such quantum optical systems of several modes in its own right.

The observation made here adds to the insight that appears to be appreciated only fairly recently, in that quantum noise does not necessarily only give rise to heating, decoherence, and dissipation, providing, in particular, a challenge in applications in quantum metrology and in quantum information science. When suitably used, quantum noise can also assist in processes thought to be necessarily of coherent nature, in noise-driven quantum phase transitions [10], quantum criticality [11], in entanglement distillation [12] or in quantum computation [13]. It turns out that thermal noise, when appropriately used, can also assist in cooling. This counterintuitive effect is not in contradiction to the laws of thermodynamics, as is plausible when viewing this setup as a thermal machine or heat engine operating in the quantum regime [14]. Unlike Ref. [15], which aims at fleshing out the smallest possible heat engine, here we ask the question whether a quantum continuous-variable system can actually be cooled with incoherent light.

The system under consideration.-We consider a system of two optical modes at frequencies $\omega_{a}$ and $\omega_{b}$, respectively, that are coupled to a mechanical degree of freedom at frequency $\omega_{c}$, Fig. 1. The Hamiltonian of the entire system is assumed to be well-approximated by $H=H_{0}+$ $H_{1}$, where the free part is given by $H_{0}=\hbar \omega_{a} a^{\dagger} a+$ $\hbar \omega_{b} b^{\dagger} b+\hbar \omega_{c} c^{\dagger} c$, and the interaction can be cast into the form

$$
H_{1}=\hbar g(a+b)^{\dagger}(a+b)\left(c+c^{\dagger}\right) \text {. }
$$

It is convenient to move to a rotating interaction picture with respect to $\hbar \omega_{b}\left(a^{\dagger} a+b^{\dagger} b\right)$. The radiation pressure interaction is invariant under this transformation, while $H_{0}$ simplifies to

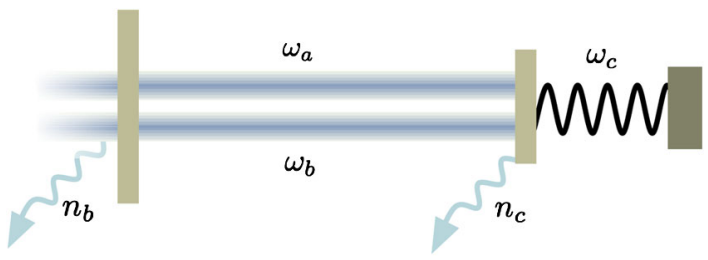

FIG. 1 (color online). The optomechanical setup primarily being considered in this work, involving two optical modes and a mechanical one. 


$$
H_{0}^{\prime}=\hbar \Delta a^{\dagger} a+\hbar \omega_{c} c^{\dagger} c,
$$

where $\Delta=\omega_{a}-\omega_{b}$. For most of what follows, the frequencies are chosen such that $\Delta=\omega_{c}$, as we will see is the optimal resonance for cooling the mechanical resonator. This can be realized by tuning the mechanical degree of freedom or the cavity mode splitting [16]. In fact, this is exactly the setting proposed in Ref. [17] as a feasible threemode optoacoustic interaction, in an idea that can be traced back to studies of parametric oscillatory instability in Fabry-Perot interferometers [18]. Similarly, with systems of high-finesse optical cavities coupled to thin semitransparent membranes [19], of double-microdisk whisperinggallery resonators [20] or of optomechanical crystals [21] such a situation can be achieved. Surely numerous other architectures are well conceivable.

In addition to this coherent dynamics, the system is assumed to undergo natural damping and decoherenceunavoidable in the optomechanical context. The quantum master equation governing the dynamics of the entire system embodying the two optical modes and the mechanical degree of freedom is given by

$$
\dot{\rho}=\mathcal{L} \rho=-\frac{i}{\hbar}[H, \rho]+\left(\mathcal{L}_{a}+\mathcal{L}_{b}+\mathcal{L}_{c}\right) \rho,
$$

with the generators being defined by $\mathcal{L}_{a}=\kappa D_{a}$ and

$$
\begin{aligned}
& \mathcal{L}_{b}=\left(1+n_{b}\right) \kappa D_{b}+n_{b} \kappa D_{b^{\dagger}}, \\
& \mathcal{L}_{c}=\left(1+n_{c}\right) \gamma D_{c}+n_{c} \gamma D_{c^{\dagger}},
\end{aligned}
$$

making use of the notation for a generator in Lindblad form

$$
D_{x}(\rho)=2 x \rho x^{\dagger}-\left\{x^{\dagger} x, \rho\right\} .
$$

The parameter $\kappa$ is the cavity decay rate, $\gamma$ is the mechanical damping rate and $n_{j}$ are the mean occupation numbers of the thermal baths of modes $j=b, c$. Here, we allow the optical bath of mode $b$ to be in a Gibbs or thermal state having an arbitrary temperature, while mode $a$ is not driven.

This type of damping reflects the plausible mechanism of loss. For the mechanical motion, we are primarily interested in the regime where $\omega_{m} \gg \gamma$, such that the damping mechanism of quantum Brownian motion based on some spectral density is virtually indistinguishable from the quantum optical Markovian damping as for an optical mode [22]. For that reason, for coherence of presentation, the same type of dissipative dynamics has been chosen for the optical and mechanical modes.

We will now discuss this given situation in two different pictures. The first one is a weak coupling approach leading to approximate analytical expressions. The second one involves sampling over colored classical stochastic processes. These methods are further discussed in the range of their validity in the EPAPS, where they are also compared with exact diagonalization methods for small photon numbers [23].

Description 1: Weak coupling approximation as an analytical approach.-In this approach, a picture is developed grasping the physical situation well for small effective couplings $g \sqrt{n_{b}}$. In addition to the actual physical baths of the three modes $a, b$, and $c$ giving rise to dissipative dynamics, we also consider mode $b$ as a further external "bath" and derive an effective master equation for modes $a$ and $c$ only. This is a good approximation if the back action on mode $b$ is negligible and up to second order in the coupling constant $g$. Having this picture in mind, the Liouvillian in Eq. (2) can be decomposed as $\mathcal{L}=\mathcal{L}_{\text {sys }}+$ $\mathcal{L}_{\text {int }}+\mathcal{L}_{\text {bath }}$, where $\mathcal{L}_{\text {bath }}=\mathcal{L}_{b}$ and

$$
\mathcal{L}_{\mathrm{sys}}=-\frac{i}{\hbar}\left[H_{0}^{\prime}, \cdot\right]+\mathcal{L}_{a}+\mathcal{L}_{c}, \quad \mathcal{L}_{\mathrm{int}}=-\frac{i}{\hbar}\left[H_{1}, \cdot\right] .
$$

Using projection operators techniques [24], one can derive a master equation for the reduced system $\rho_{a, c}=\operatorname{tr}_{b}[\rho]$

$$
\begin{aligned}
\dot{\rho}_{a, c}(t)= & \mathcal{L}_{\mathrm{sys}} \rho_{a, c}(t) \\
& +\operatorname{tr}_{b} \mathcal{L}_{\mathrm{int}} \int_{0}^{\infty} d s e^{\mathcal{L}_{r} s} \mathcal{L}_{\mathrm{int}} \rho_{a, c}(t-s) \otimes \rho_{b} .
\end{aligned}
$$

Here $\mathcal{L}_{r}=\mathcal{L}_{\text {sys }}+\mathcal{L}_{\text {bath }}$ and $\mathcal{L}_{b} \rho_{b}=0$. Making use of the explicit expression (6) for $\mathcal{L}_{\text {int }}$, we have

$$
\begin{aligned}
\dot{\rho}_{a, c}(t)= & \mathcal{L}_{\mathrm{sys}} \rho_{a, c}(t) \\
& -\frac{1}{\hbar^{2}} \operatorname{tr}_{b}\left[H_{1}, \int_{0}^{\infty} d s e^{\mathcal{L}_{r} s}\left[H_{1}, \rho_{a, c}(t-s) \otimes \rho_{b}\right]\right] .
\end{aligned}
$$

In what follows, we will make a sequential approximation of the interaction Hamiltonian $H_{1}$ and the damping mechanism. In order to be as transparent as possible, we mark each of the steps with a roman letter.

Equation (7) - up to second order expansion in the coupling $g$, which constitutes the first approximation step (a) — can also be written as

$$
\begin{aligned}
\dot{\rho}_{a, c}(t)= & \mathcal{L}_{\mathrm{sys}} \rho_{a, c}(t) \\
& -\frac{1}{\hbar^{2}} \operatorname{tr}_{b}\left[H_{1}, \int_{0}^{\infty} d s\left[e^{\mathcal{L}_{r}^{\dagger} s}\left(H_{1}\right), \rho_{a, c}(t) \otimes \rho_{b}\right]\right],
\end{aligned}
$$

where $\mathcal{L}_{r}^{\dagger}$ acts only on the Hamiltonian $H_{1}$, corresponding to a "dissipative interaction picture" with respect to $\mathcal{L}_{r}$.

We start from Eq. (1) and (b) neglect the term proportional to $a^{\dagger} a$ because we assume mode $a$ to be weakly perturbed from its ground state. In contrast, we allow the physical optical bath of mode $b$ to have an arbitrary temperature and therefore we cannot neglect the term proportional to $b^{\dagger} b$. We rewrite the approximated $H_{1}$ as

$$
H_{1}^{\prime}=\hbar g\left(a^{\dagger} b+b^{\dagger} a+\delta\right)\left(c+c^{\dagger}\right),
$$


where the operator $\delta=b^{\dagger} b-n_{b}$ represents the intensity fluctuations of mode $b$. In order to have vanishing first moments with respect to mode $b$, the mean force proportional to $\left\langle b^{\dagger} b\right\rangle$ has been subtracted, which is responsible of merely shifting the resonator equilibrium position. Since $\omega_{a}-\omega_{b}=\omega_{c}$, the (c) rotating wave approximation (RWA) of Eq. (9) is

$$
H_{1}^{\prime \prime}=\hbar g\left(a^{\dagger} b c+a b^{\dagger} c^{\dagger}\right)+\hbar g \delta\left(c+c^{\dagger}\right) .
$$

As will be explained later in more details, the first term of the Hamiltonian is responsible for the cooling of the mechanical resonator, while the second term corresponds to an additional heating noise.

In order to compute the partial trace in Eq. (8), we need the two-time correlation functions of the thermal light in mode $b$,

$$
\left\langle b e^{\mathcal{L}_{r}^{\dagger} s} b^{\dagger}\right\rangle=e^{-\kappa s} n_{b}, \quad\left\langle\delta e^{\mathcal{L}_{r}^{\dagger} s} \delta\right\rangle=e^{-2 \kappa s}\left(n_{b}^{2}+n_{b}\right) .
$$

The exponential functions in Eqs. (11) determine the time scale of the integral kernel in Eq. (8), which will be of the order of $\kappa^{-1}$. Within this time scale (d) we can neglect the effect of the mechanical reservoir $(\gamma \ll \kappa)$, and the action of the map $e^{\mathcal{L}_{r}^{\dagger} s}$ on the system operators will be

$$
\begin{aligned}
& e^{\mathcal{L}_{r}^{\dagger} s} a=e^{-(\kappa+i \Delta) s} a=e^{-\left(\kappa+i \omega_{c}\right) s} a, \\
& e^{\mathcal{L}_{r}^{\dagger} s} c=e^{-\left(\gamma+i \omega_{c}\right) s} c \simeq e^{-i \omega_{c} s} c .
\end{aligned}
$$

We can finally perform the integration in Eq. (8), and since all the odd moments of $\rho_{b}$ vanish, the cooling and heating terms in Eq. (10) generate two independent contributions to the master equation, respectively

$$
\begin{gathered}
\mathcal{L}_{\text {cool }}=\frac{g^{2}}{2 \kappa}\left(\left(1+n_{b}\right) D_{a c^{\dagger}}+n_{b} D_{a^{\dagger}}\right), \\
\mathcal{L}_{\text {heat }}=\frac{2 \kappa g^{2}\left(n_{b}^{2}+n_{b}\right)}{4 \kappa^{2}+\omega_{c}^{2}}\left(D_{c^{\dagger}}+D_{c}\right),
\end{gathered}
$$

where in calculating $\mathcal{L}_{\text {heat }}$ we (e) kept only the counterrotating terms. The effect of $\mathcal{L}_{\text {heat }}$ is simply a renormalization of the mean occupation number of the mechanical bath

$$
n_{c} \mapsto \tilde{n}_{c}=n_{c}+\frac{2 \kappa g^{2}\left(n_{b}^{2}+n_{b}\right)}{\gamma\left(4 \kappa^{2}+\omega_{c}^{2}\right)},
$$

always increasing, as expected, the effective temperature of the environment. Denoting with $\tilde{\mathcal{L}}_{\text {sys }}$ the corresponding renormalized Liouvillian, the master equation can be written as

$$
\dot{\rho}_{a, c}=\left(\tilde{\mathcal{L}}_{\mathrm{sys}}+\mathcal{L}_{\mathrm{cool}}\right) \rho_{a, c} .
$$

With respect to Eq. (2) and (14), can be numerically solved with much less computational resources but we have to remind ourselves that this approach is valid only within the
RWA and for weak coupling: $\gamma, g \ll \omega_{c}$. Another advantage of Eq. (14) is that the corresponding adjoint equations for the number operators $\hat{n}_{a}=a^{\dagger} a$ and $\hat{n}_{c}=c^{\dagger} c$ are closed with respect to these operators, that is

$$
\begin{aligned}
& \dot{\hat{n}}_{a}=-2 \kappa \hat{n}_{a}-\frac{g^{2}}{\kappa}\left(\left(n_{b}+1\right) \hat{n}_{a}-n_{b} \hat{n}_{c}-\hat{n}_{a} \hat{n}_{c}\right), \\
& \dot{\hat{n}}_{c}=-2 \gamma \hat{n}_{c}-\frac{g^{2}}{\kappa}\left(n_{b} \hat{n}_{c}-\left(n_{b}+1\right) \hat{n}_{a}-\hat{n}_{a} \hat{n}_{c}\right)+2 \gamma \tilde{n}_{c} .
\end{aligned}
$$

Assuming $(f)$ that the factorization property $\left\langle\hat{n}_{a} \hat{n}_{c}\right\rangle \simeq$ $\left\langle\hat{n}_{a}\right\rangle\left\langle\hat{n}_{c}\right\rangle$ holds-which is essentially a mean-field approach which is expected to be good in case of small correlations, or, again as assumed, for small values of $g$-we can find analytical expressions for the steady state expectation values:

$$
\begin{aligned}
& \left\langle\hat{n}_{c}\right\rangle=\frac{\tilde{n}_{c}-\eta}{2}+\left(\frac{\left(\tilde{n}_{c}+\eta\right)^{2}}{4}-\frac{\kappa n_{b} \tilde{n}_{c}}{\gamma}\right)^{1 / 2}, \\
& \left\langle\hat{n}_{a}\right\rangle=\frac{\left(\tilde{n}_{c}-\left\langle\hat{n}_{c}\right\rangle\right) \gamma}{\kappa},
\end{aligned}
$$

where $\eta=1+n_{b}(1+\kappa / \gamma)+2 \kappa^{2} / g^{2}$.

Description 2: Sampling with respect to colored stationary classical stochastic processes.-In this approach, we start from the exact dynamics Eq. (2) but treat mode $b$ as a classical thermal field and neglect any feedback from the resonator. We substitute the bosonic operator with a complex amplitude $b(t) \mapsto \beta_{t}$, giving rise to a semiclassical picture. The parameter $\beta_{t}$ can be described as a classical stochastic process defined by the stochastic differential equation (SDE)

$$
d \beta_{t}=-\kappa \beta_{t} d t+\sqrt{\kappa n_{b}}\left(d W^{(x)}+i d W^{(y)}\right),
$$

with independent Wiener increments [9] obeying the Itō rules $d W^{(a)} d W^{(b)}=\delta_{a, b} d t, d W^{(a, b)} d t=0$. The dynamics of the remaining modes $a$ and $c$ instead, can be efficiently treated quantum mechanically; this is true, since for every single realization of the process (15), the evolution defines a Gaussian completely positive map and therefore the corresponding Gaussian state $\rho_{a, c}^{\left(\beta_{t}\right)}(t)=\mathcal{E}_{t}^{\left(\beta_{t}\right)}\left(\rho_{a, c}\right)$ can be described entirely in terms of first and second moments. The actual quantum state of the system will in general not be exactly Gaussian, it can nonetheless be simulated by sampling over many Gaussian states associated with different realizations of $\beta_{t}$ : Only the respective weight in the convex combinations are such that the resulting state can be non-Gaussian. The resulting state $\rho_{a, c}(t)=\mathbb{E} \rho_{a, c}^{\left(\beta_{t}\right)}(t)$ will be our semiclassical description of the system.

It is convenient to introduce a vector of quadratures operators $u=\left[x_{c}, y_{c}, x_{a}, y_{a}\right]$, where $x_{j}=\left(j+j^{\dagger}\right) / \sqrt{2}$, $y_{j}=i\left(j^{\dagger}-j\right) / \sqrt{2}$ and $j=a, c$. From Eq. (2), we get a SDE for the first moments

$$
\frac{d\langle u\rangle_{t}}{d t}=A_{t}\langle u\rangle_{t}+f_{t}
$$


where

$$
A_{t}=\left[\begin{array}{cccc}
-\gamma & \omega_{c} & 0 & 0 \\
-\omega_{c} & -\gamma & g \beta_{t}^{(x)} & g \beta_{t}^{(y)} \\
-g \beta_{t}^{(y)} & 0 & -\kappa & \Delta \\
g \beta_{t}^{(x)} & 0 & -\Delta & -\kappa
\end{array}\right], \quad f_{t}=\left[\begin{array}{c}
0 \\
g\left|\beta_{t}\right|^{2} \\
0 \\
0
\end{array}\right],
$$

$\beta_{t}^{(x)}=\left(\beta_{t}+\beta_{t}^{*}\right), \quad \beta_{t}^{(y)}=i\left(\beta_{t}^{*}-\beta_{t}\right)$. The second moments can be arranged in the matrix $V_{t}=\operatorname{Re}\left\langle u u^{\dagger}\right\rangle_{t}$, satisfying the SDE

$$
\frac{d V_{t}}{d t}=A_{t} V_{t}+V_{t} A_{t}^{T}+D+F_{t}
$$

where $D=\operatorname{diag}\left[\gamma\left(2 n_{c}+1\right), \gamma\left(2 n_{c}+1\right), \kappa, \kappa\right]$, and $F_{t}=$ $f_{t}\langle u\rangle_{t}^{T}+\langle u\rangle_{t} f_{t}^{T}$. The statistical average over many realization of $V_{t}$ will be an estimator for the second moments of the quantum state $V(t)=\mathbb{E}\left(V_{t}\right)$. In particular, the first two diagonal elements give the effective phonon number of the mechanical oscillator, since $\left\langle n_{c}\right\rangle(t)=\left(V_{1,1}(t)+\right.$ $\left.V_{2,2}(t)-1\right) / 2$. The three stochastic differential Eqs. (15), (16), and (18) can be numerically integrated in sequential order. In our simulations, see Fig. 2, we used the Euler method, for each time step $d t$ sampling the associated Wiener increments in Eq. (15) with normal distributions of variance $\sigma^{2}=d t$.

Intuitive explanation of the effect of cooling by heating.-This effect can be intuitively explained at hand of Eq. (10) in Description 1: Two competing processes play here an important role: The first term appears like a beam splitter interaction between the modes $a$ and $c$ with a "reflectivity" given by the thermal fluctuations of the

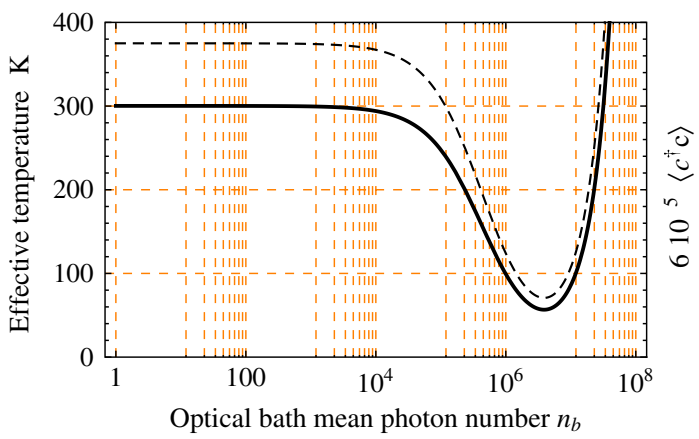

FIG. 2 (color online). Room temperature cooling with parameters reminding of those typical in realistic experiments [3]: $\omega_{c}=$ $2 \pi \mathrm{MHz}, \kappa=0.2 \omega_{c}, g=0.3 \times 10^{-5} \omega_{c}$, and $\gamma=10^{-3} \omega_{c}$. The black line shows the predictions of the steady state using Description 1, the dots are a result from stochastic sampling using Description 2 (with 100 realizations), which qualitatively coincide well. The dashed line shows the expected phonon number scaled by a factor of $6 \times 10^{-5}$. One clearly finds that an increased population of mode $b$ leads to a significant cooling of the mechanical mode-up to a point when eventually the "bad noise" becomes dominant. amplitude of mode $b$. This is responsible for the cooling of the mirror. That is to say, the occupation of mode $b$ takes the role resembling the "basis of a transistor": A high occupation renders the interaction between $a$ and $c$ stronger, hence triggering the cooling effect. For this effect to be relevant, the coherence or purity of the state of $b$ does not play a dominant role, and hence even thermal noise can give rise to cooling. This is referred to as "good noise". The second term corresponds to the fluctuations of the radiation pressure of mode $b$ and it is a source of "bad noise" which heats the mechanical mode.

Similarly, this effect can be studied at hand of the stochastic picture of Description 2, when observing Eq. (18). In addition to the intrinsic quantum noise described by $D$, stochastic fluctuations of $\beta_{t}$ generate an additional heating noise given by the matrix $F_{t}$. However, the same process $\beta_{t}$ is also contained in the matrix $A_{t}$ and corresponds to a cooling noise, up to the approximations identical to the above "good noise." The reason is quite evident from Eq. (17), where we observe that the coupling between the hot mechanical oscillator and the cold optical mode is mediated by the thermal fluctuations of $\beta_{t}$. This optomechanical coupling, which would be zero without noise, leads to a sympathetic cooling of the mechanical mode.

Example.-We will now discuss the effect of cooling by heating at hand of an example using realistic parameters in an optomechanical setting. Figure 2 shows the effective temperature of the mechanical mode as a function of the number of photons in mode $b$ : Here, effective temperature is defined as the temperature $T$ of a Gibbs state

$$
\rho_{c}(T)=\frac{e^{-\hbar \omega_{c} c^{\dagger} c /(k T)}}{\operatorname{tr}\left(e^{-\hbar \omega_{c} c^{\dagger} c /(k T)}\right)}
$$

such that $\left\langle\hat{n}_{c}\right\rangle=\operatorname{tr}\left(\rho_{c}(T) c^{\dagger} c\right)$. One quite impressively encounters the effect of cooling by heating, for increasing photon number and hence effective temperature of this optical mode. For very large values of the photon number, the "bad noise" eventually becomes dominant, resulting again in a heating up of the mechanical mode. Note that needless to say, the effective temperature of the optical mode $b$ is usually larger than the mechanical one by many orders of magnitude (approximately $10^{10} \mathrm{~K}$ for reasonable parameters).

Summary.-In this work, we have established the notion of cooling by heating, which means that cooling processes can be assisted by means of incoherent hot thermal light. We focused on an optomechanical implementation of this paradigm. We also introduced new theoretical tools to grasp the situation of driving by classical noise, including sampling techniques over stochastic processes. To experimentally demonstrate this counterintuitive effect should be exciting in its own right. Putting things upside down, one could also conceive settings similar to the one discussed here as demonstrators of small heat engines [15] operating 
at the quantum mechanical level, where $b$ takes the role of an "engine" and mode $a$ of a "condenser". To fully explore these implications for feasibly realizing quantum thermal machines constitutes an exciting perspective. It would also be interesting to fully flesh out the potential for the effect to assist in generating nonclassical states [25] "the bit" or entanglement $[5,26]$. Finally, quite intriguingly, this work may open up ways to think of optically cooling mechanical systems without using lasers at all, but rather with basic, cheap LEDs emitting incoherent light.

We would like to thank the EU (Minos, Compas, Qessence), the EURYI, and QuOReP for support.

[1] S. Gigan et al., Nature (London) 444, 67 (2006); O. Arcizet et al., ibid. 444, 71 (2006); D. Kleckner and D. Bouwmeester, ibid. 444, 75 (2006).

[2] A. Schliesser et al., Nature Phys. 4, 415 (2008).

[3] S. Groeblacher et al., Nature Phys. 5, 485 (2009); Nature (London) 460, 724 (2009).

[4] D. Vitali, S. Mancini, L. Ribichini, and P. Tombesi, Phys. Rev. A 65, 063803 (2002).

[5] A. Ferreira, A. Guerreiro, and V. Vedral, Phys. Rev. Lett. 96, 060407 (2006); M. Paternostro et al., ibid. 99, 250401 (2007); D. Vitali et al., Phys. Rev. Lett. 98, 030405 (2007); C. Genes et al., Phys. Rev. A 78, 032316 (2008).

[6] F. Marquardt and S. M. Girvin, Physics 2, 40 (2009).

[7] This cooling is not due to some energy minimum being favored, with a transition facilitated by small amounts of quantum noise, reminding of quantum versions of stochastic resonance [8].

[8] M. B. Plenio and S. F. Huelga, Phys. Rev. Lett. 88, 197901 (2002); I. Goychuk and P. Hänggi, ibid. 91, 070601 (2003).

[9] N. G. van Kampen, Stochastic Processes in Physics and Chemistry (Elsevier, New York, 2007).

[10] S. Diehl, A. Micheli, A. Kantian, B. Kraus, H. P. Büchler, and P. Zoller, Nature Phys. 4, 878 (2008).
[11] J. Eisert and T. Prosen, arXiv:1012.5013.

[12] K. G. H. Vollbrecht, C. A. Muschik, and J. I. Cirac, Phys. Rev. Lett. 107, 120502 (2011).

[13] F. Verstraete, M. M. Wolf, and J. I. Cirac, Nature Phys. 5, 633 (2009).

[14] J. Gemmer, M. Michel, and G. Mahler, Quantum Thermodynamics, Lecture Notes in Physics Vol. 784 (Springer, Heidelberg, 2009).

[15] N. Linden, S. Popescu, and P. Skrzypczyk, Phys. Rev. Lett. 105, 130401 (2010); S. Popescu, arXiv:1009.2536.

[16] The modes can be considered as independent in the resolved sideband limit $\omega_{c}<\kappa$ (which is the regime studied here). In this regime the two modes are coupled with two distinct frequency windows of the bath and in this sense we can model them with two independent baths.

[17] Z.-Q. Yin, Phys. Rev. A 80, 033821 (2009); H. Miao et al., Phys. Rev. A 78, 063809 (2008); C. Zhao et al., Phys. Rev. Lett. 102, 243902 (2009).

[18] V. B. Braginsky, S. E. Strigin, and S. P. Vyatchanin, Phys. Lett. A 287, 331 (2001).

[19] C. Biancofiore et al., Phys. Rev. A 84, 033814 (2011).

[20] X. Jiang et al., Opt. Express 17, 20911 (2009).

[21] T.P. Mayer Alegre, A. Safavi-Naeini, M. Winger, and O. Painter, Opt. Express 19, 5658 (2011).

[22] A picture of quantum Brownian motion has also been compared to this quantum optical Markovian limit, with no notable differences in any predictions within the parameters that are of interest in this work.

[23] See supplemental material at http://link.aps.org/ supplemental/10.1103/PhysRevLett.108.120602 for details.

[24] C. W. Gardiner and P. Zoller, Quantum Noise (Springer, New York, 2004).

[25] A. Mari and J. Eisert, Phys. Rev. Lett. 103, 213603 (2009); K. Jaehne, C. Genes, K. Hammerer, M. Wallquist, E. S. Polzik, and P. Zoller, Phys. Rev. 79, 063819 (2009); A. A. Clerk, F. Marquardt, and K. Jacobs, New J. Phys. 10, 095010 (2008).

[26] J. Eisert, M. B. Plenio, S. Bose, and J. Hartley, Phys. Rev. Lett. 93, 190402 (2004); I. Wilson-Rae, P. Zoller, and A. Imamoglu, ibid. 92, 075507 (2004). 